\title{
Cloud Computing to Support E-Learning and Business in SMEs
}

\author{
Ileana Hamburg ${ }^{1}$, Emma O Brien ${ }^{2}$ \\ Institut Arbeit und Technik (IAT) - WH Gelsenkirchen ${ }^{1}$, Germany \\ University of Limerick ${ }^{2}$, Limerick, Ireland
}

\begin{abstract}
SMEs contribute to economic growth in Europe. The financial crisis and the economic recession have hit SMEs hard in the EU28 and the economic conditions remain difficult. The potential for cloud computing to support business as a platform for $E$ Learning is significant. These technologies can be adapted over time to scale to the required resources. This paper will examine the benefits and limitations of cloud computing to support business and in particular workplace learning in SMEs, It will examine the barriers to adoption and outline the work of projects conducted in this area by the authors.
\end{abstract}

\section{Introduction}

Cloud computing offers many opportunities including digital learning, it can help companies to improve their business and use technology more efficiently.

The NIST definitions of Cloud Computing (National Institute of Standards and Technology) are used as a starting point for the discussion in this paper "Cloud computing is a model for enabling convenient, on-demand network access to a shared pool of configurable computing resources (e.g., networks, servers, storage, applications, and services) that can be rapidly provisioned and released with minimal management effort or service provider interaction" [1].

In addition Marston et al. define cloud computing as "an information technology service model where computing services (both hardware and software) are delivered on-demand to customers over a network in a self-service fashion, independent of device and location" [2]. The resources required to provide the requisite quality-of service levels are shared, dynamically scalable, rapidly provisioned, virtualized and released with minimal service provider interaction. Users pay for the service as an operating expense without incurring any significant initial capital expenditure, with the cloud services employing a metering system that divides the computing resource in appropriate blocks".
Agility, elastic scalability, low costs are some benefits of using cloud computing; data can be moved smoothly without boundaries. Until now mostly big corporations availed of the advantages of cloud technologies. SMEs are reluctant to consider this technology and the adoption of cloud computing in SMEs is hindered due to lack of awareness with this subject and the absence of guidelines and best practices [3].

SMEs contribute to economic growth in Europe. In the EU28, in 2013 some 21.6 million SMEs employed 88.8 million people and generated 3.666 trillion in values added [4]. The financial crisis and the economic recession have hit SMEs hard in the EU28 and the economic conditions remain difficult. Many of these companies are micro enterprises with few resources; they face technological, economic and financial difficulties. Changes in ICT infrastructure require more resources, often only for a short period of time. SMEs have to be convinced that the use of cloud computing can help them to avoid over proportioning ICT infrastructures and reduce costs. Cloud services allow SMEs to avail of providers which offer them resources based on pay-as-you go and subscription models eliminating the need to own and maintain ICT hardware [5].

Laugesen et al. highlighted that few studies have been conducted on the ICT skills required for SMEs to become more competitive [6]. The economic pressure is a threat to continuous training and learning.

Often the lack of knowledge and skills in this area can be a barrier to adopting cloud computing [7]. In addition Laugesen et al. found that many training offerings are very technical by nature and not relevant to the business needs of the organization. E-Learning is suitable for SMEs to achieve competitive skills due to its flexibility in content, time and place but is not sufficiently used in SMEs.

Cloud computing can be adapted to support E-Learning in SMEs as it a natural platform which can be scaled over time to offer the necessary resources.

The paper will look at the benefits and limitations of cloud computing to support business and its 
ability to facilitate increased learning activities in SMEs by addressing the barriers to adoption they face. given.

Examples of projects involving the authors are

\section{Business and Learning Methods Used in SMEs}

2013 could be considered as a turning point for European SMEs [4]. After years of an uncertain economic situation, 2013 is first year that there has been a combined increase in employment and valueadded of European SMEs.

The figure 1 shows the degree of recovery of SMEs from 2008 to 2013.

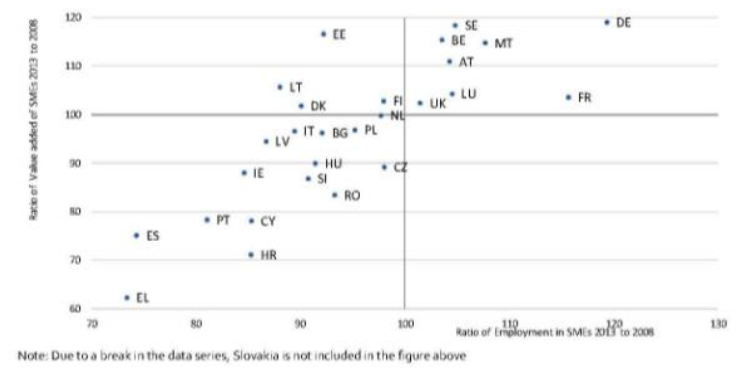

Figure 1. SME Degree of Recovery from 2008 to 2013, Value Added and Employment

It is known that the current business SME environments are characterized by increasing competition and the day to day pressure of running an efficient enterprise. Low demand for the goods and services, which SME produce, explains why SME performance did not recover to pre-recession levels in some member states.

The most important SME sectors are wholesale and retail sector and the largest ones are manufacturing, construction, accommodation and foods. In 2013 positive growth was reported in business services, retail and wholesale trade; the construction industry has suffered severely.

Many SMEs are not in export-oriented sectors, particularly the micro and small enterprises.

Generally many SMEs are struggling to survive in an ongoing global recession and often they are becoming reluctant to release or pay for staff training which can contribute to the difficulty in recovering this situation.

Research has highlighted the role of training and skills development on business success [8] [9] [10] [11] [12]. However, in difficult times training budgets are often the first costs reduced or removed. This is often because owner/managers of SMEs do not have enough knowledge about long term value of training in sustaining competitive advantage.
The predominant training method for SMEs is workplace training as it is viewed as 'low cost' [13] [14]. The integration with formal strategically aligned training is often not planned.

The current informal forms of training used in SMEs have a positive impact with regard to productivity, but informal learning alone is not a creative solution and not always cheap, particularly when it does not align with the company's strategy and business requirements.

E-Learning has tried to address issues of time and cost, by allowing employees to access learning resources remotely. The learning material is easy to keep updated, the trainers can integrate multimedia content which facilitates the understanding and motivates the participants, but this form of learning is not used. Blended learning, combining the positive aspects of teacher-driven learning which is usual in many SMEs with e-Learning, is a suitable way to learn in SMEs and increases their competitiveness often not implemented.

Obstacles for this are the costs of development, of delivery, time and available budget. Additionally, many SMEs do not have a dedicated human resources person or even an employee that can be charged with the responsibility of searching for relevant learning resources and needs.

Cloud computing has the potential to improve education and particularly e-Learning in SMEs. Cloud can be viewed as Education Software as a Service.

Managers are not always aware of the importance of new technologies, of using other forms of learning like problem-based learning, mobile learning, webinars, access to on-demand learning resources and social learning supported by social media for up to date skills and information.

They need to assess the impact of such forms of learning and platforms within the SMEs business and different organizational contexts. Learning objectives as well as organizational drivers must be considered [15].

\section{Cloud Computing to Support Business in SMEs}

The main characteristics of Cloud Computing which are also useful for SMEs are [1] [16]:

- On-demand self-service. The user can benefit from capabilities, such as server time and network storage, without requiring human interaction with each service provider.

- Broad network access. Capabilities are available over the network and accessed through standard mechanisms

- Resource pooling. The resources are pooled to serve multiple users who generally have no control or knowledge over the exact location. 
Examples of resources include storage, processing, memory, network bandwidth, and virtual machines.

- Rapid elasticity. Capabilities can be rapidly and elastically provisioned

- Measured Service. Resource usage can be monitored, controlled, and reported providing transparency for both the provider and user of the utilized service.

Figure 2 shows the most deployed models of Cloud Computing. They define the type of access to the cloud, i.e., how the cloud is located? Cloud can have any of the four types of access: Public, Private, Hybrid and Community.

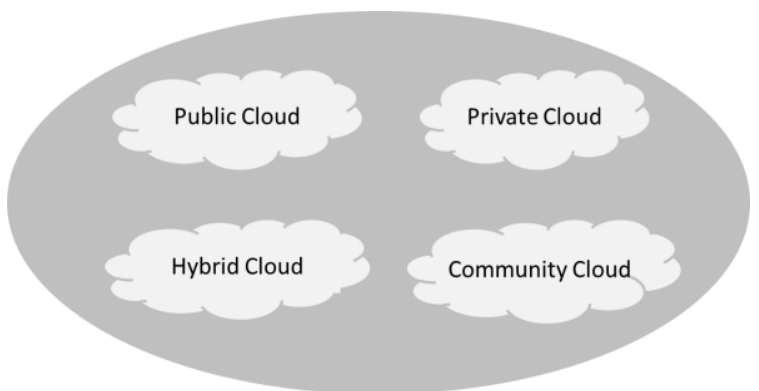

Figure 2. Models of Cloud

Service Models are the reference models on which the Cloud Computing is based. These can be categorized into three basic service models as listed below [1]:

- Cloud Software as a service (SaaS). Provider's applications running on a cloud infrastructure are accessible from various client devices through a thin client interface such as a web browser.

- Cloud Platform as a service (PaaS). The user develops on the cloud infrastructure or acquires applications created using programming languages and tools supported by the provider.

- Cloud Infrastructure as a service (IaaS). The CSP provides the user with storage, networks, and other fundamental computing resources where the consumer is able to deploy and run arbitrary software, which can include operating systems and applications.

By using Cloud services SMEs can avail of opportunities which allow them to compete in an innovative ICT environment, and give a level playing field required to succeed in business [17]. SMEs can grow their business without the need for a new equipment or infrastructure that gets outdated easily.

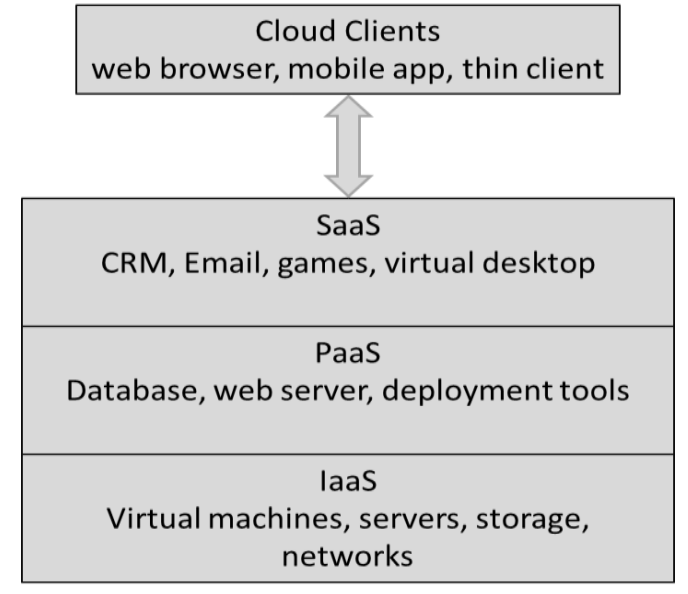

Figure 3. Service Models

"They don't want to manage that equipment any more, it's just too intensive from a resource perspective and from a monetary perspective, and it's inflexible, as it won't adapt itself to companies during a time of expansion. With an on-demand infrastructure you can start with what you need today without worrying about tomorrow or the day after because the cloud can scale with you as and when needed", Bryce said.

Because many SMEs are oriented to high-speed network connections cloud technology allows them to take advantages of hosted services.

Some advantages of the Cloud for SMEs are [3] [17] [18]:

- Up-to-date software solutions without placing too much cost on the business.

- Availability of unlimited data storage, which can be expanded anytime.

- Access to data from anywhere and anytime means portability and flexibility; giving more time and effort to be placed on business strategies and solutions.

- Sophisticated and high levels of security protocol that ensure business and data protection.

- Better business performance due to the portability, flexibility, efficiency and productivity that the cloud provides.

- Simplified back-end data management and control using automatic cross referencing and reconciliation.

With any technology there are also a number of limitations or issues with cloud computing. One of the main issues is the reliability and security of data and the accessibility of this on a 24/7 basis, particularly when the cloud service provider has an outage. Many companies will have problems about the lack of control over their ICT systems and the impact of a CSP on these [3]. These issues may inhibit a SMEs decision to migrate to a cloud 
computing environment. In addition there are other factors which may influence the decision [1] [9]:

- The lack of understanding of the infrastructure, cost and appropriateness to the needs and scenarios of different companies from different business environments

- The ICT skill levels of users, managers and entrepreneurs

- The readiness of SMEs to adopt cloud computing from a business perspective

- Lack of time

Some of these issues can be addressed by educating employees on the concept of cloud computing and developing business based ICT skills in SMEs. This will allow them to make informed decisions about the appropriateness of cloud computing to their business strategy and what aspects can benefit them the most.

\section{Cloud Computing to Support E-learning in SMEs}

Some aspects which could be improved by using could computing to implement e-learning are poor scalability at the infrastructure level, development and assigning of resources only for determined tasks, need to configure and add new resources making the costs and resource management very expensive [20] [21].

Two main characteristics of Cloud computing which could be an alternative of traditional ICT centers and could improve the E-Learning approaches in SMEs are the use of resources "on demand" and the transparent scalability so that the computational resources are assigned dynamically when they are necessary without the necessity of infrastructure understanding by the users.

Cloud computing supports the efficient utilization of E-Learning resources following a dynamic rule of use. Costs related to computer maintenance disappear.

Masud and Huang pointed some consequences and implications when the E-Learning services are deployed using Cloud computing environments [22]:

- Accessed via Web

- Subscribers do not pay for installation, software maintenance, deployment and server administration

- Pay by subscription based on usage

- Very high of security should be given by Cloud providers because subscriber data are held on SaaS server

Ouf et al. underplayed potential values of Cloud computing like the following [23]:
- When client computer crashes, there are almost no data lost because everything is stored in the cloud

- Students can work from different places, can find their files and edit them through the cloud and browser-based applications accessed through various devices

- Virtualization which makes possible the rapid replacement of a cloud located server

- Centralized data storage

- Easier monitoring of data access

Figure 4 shows the architecture of a Cloud computing platform for E-Learning which is usually common to most E-Learning approaches on the Cloud. (Source [24])

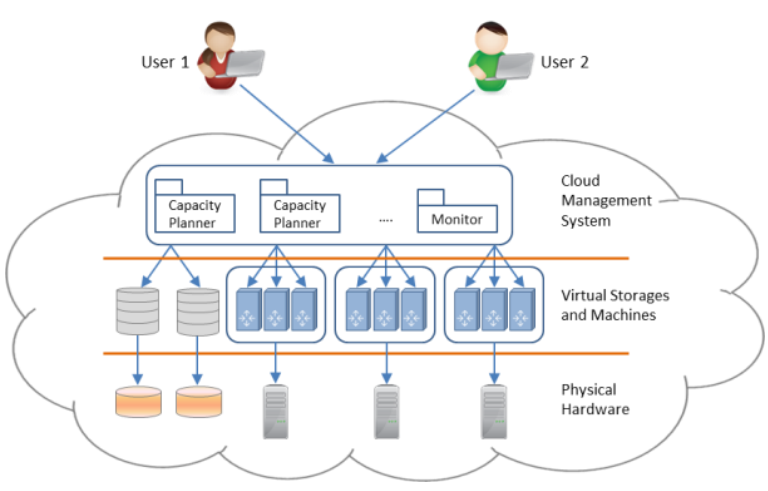

Figure 4. The Architecture of a Cloud Computer Platform for E-Learning

E-Learning could be used also to develop ICT skills in cloud computing for SMEs.

Few studies have been conducted into the emerging ICT skills required for cloud computing. Much research has focused on the technological aspects of cloud computing rather than the skills and preparation companies required to allow them avail of the strategic benefits this technology offers. In Laugesen et al. conducted an in-depth study of such skills with 72 companies, experts, public sector representatives and representative institutions on eskills requirements for cloud computing [6]. They found that the focus from primarily technical skills has moved to business skills primarily those that are concerned with enabling and managing ICT as well as strategy development and managing change. Skills in the security of information were viewed as one of the most important. They also investigated the courses available to satisfy these skills needs and found that they were very technical, slow moving and only covered partial elements. In addition there were few courses that were 'vendor neutral'. Thus it is important that contextualized business related ICT skills are developed simultaneously with technology that informs companies of the range of technologies 
available out there and how they can assist with competitive advantage.

\section{Examples and Conclusions}

The European project Net Knowing 2.0 aimed at improving E-Learning, knowledge management and use of new technologies in SMEs. In order to research the needs and problems the German SMEs have in adopting cloud technology, some interviews were conducted by the authors with managers from SMEs. After the analysis of the interviews it became clear that scenarios for the introduction of cloud computing in SMEs following the results of the interviews were necessary [25].

Another project in which the authors use scenarios is SmartPA (www.smartpa.eu). Within the project SmartPA, mentors will be trained for accountants and staff from public administrations to use cloud services [20].

The EU Erasmus+ project, supporting problem based learning in SMEs through IT facilitated mentoring - Archimedes - will develop a framework for organisational problem-based learning and supports the use of this form of learning which should be widely adopted in SMEs such as informal/social learning. It will be realised through formal and informal mentoring processes. The project will use e-Learning content and encouraging the use of cloud computing and ICT based social networking [25].

As a conclusion from literature research and projects it is evident that the advantages of Cloud computing to support business and learning in SMEs are not thoroughly explored.

This paper explained some advantages in the use of Cloud Computing such as flexibility and scalability. It highlights its ability to optimize ICT resource management in SMEs can often be a big problem.

\section{Acknowledgements}

This paper describes work within the European projects Net Knowing 2.0 and SmartPA, within the Lifelong Learning Program and on-going Erasmus+ project Archimedes.

\section{References}

[1] National Institute of Standards and Technology, "The NIST Definition of Cloud Computing (draft)", Online January 2011: http://csrc.nist.gov/publications/drafts/ 800-145/Draft-SP-800-145_cloud-definition.pdf, 2011.

[2] Marston, S., Li, Z., Bandyopadhyay, S., Zhang, J. and Ghalsasi, A., "Cloud computing - The business perspective", Decision Support Systems 51: pp. 176-189, 2011.
[3] Hamburg, I. and Marian, M., "Supporting knowledge transfer and mentoring in companies by e-learning and cloud computing", ICWL 2012 International Workshops, KMEL, SciLearn, and CCSTED, Sinaia, Romania, September 2-4, 2012; revised selected papers. Heidelberg: Springer: pp. 231-240.

[4] European Commission, "A recovery on the horizon?" Annual report on European SMEs, 2013.

[5] Hamburg, I. and Marian, M., "Knowledge transfer and mentoring in companies by e-learning and cloud computing", Chiu, D., Popescu E., Li, Q. (eds.): ICWL 2012: the 11th International Conference on Web-based Learning; 02.-04.09.2012, Sinaia, Romania; local workshop proceedings. Craiova: Univ. of Craiova: pp. 91101, 2012.

[6] Laugesen, N., Lauritzen, J.R., Carpenter, G., Ellegaard, C.M., Bucher, M. and Stabe, M., "Cloud Computing Cyber Security and Green IT The impact on e-Skills requirements", Prepared by the Danish technological institute and Fraunhofer for the European Commission, May 2012.

[7] Carcary, M., Doherty, E. and Conway, G., "Understanding and Supporting Cloud Computing Adoption in Irish Small- and Medium-Sized Enterprises", $A$ white paper by the innovation value institute, September 2013.

[8] Blundell, R., Dearden, L., Meghir, C. and Sianesi, B., "Human Capital Investment: The Returns from Education and Training to the Individual, the Firm and the Economy",, Fiscal Studies 20(1): 1-23, 1999.

[9] Ashton, D.N. and Felstead, A., "Training and Development", Human Resource Management: A Critical Text, S. J. London, Routledge, 1995.

[10] Holzer, H., Block, M. and Knott, J., "Are Training Subsidies for firms Effective? The Michigan Experience." Industrial and Labor Relations Review 46(4): pp. 625 636, 1993.

[11] Conti, G., "Training Productivity and Wages", The sixteenth annual European Association of Labour Economists (EALE), Lisbon, Portugal, 2004.

[12] Black, S. and Lynch, L., "Human Capital Investments and Productivity", American Economic Review 1996 (May): pp. 263-267, 1996.

[13] Kok, J., Vroonhof, P., Verhoeven, W., Timmermans, N., Kwaak, T., Snijders, J. and Westhof, F., "Do SMEs create more and better jobs?", EIM Business \& Policy Research with financial support from the European Communities, under the Competitiveness and Innovation Programme, 2011.

[14] Admiraal, W.and Lockhorst, D., "E-Learning in Small and Medium-sized Enterprises across Europe : Attitudes towards Technology, Learning and Training", International Small Business Journal (27): p. 743, 2009

[15] Jones, P., "What's the Point? Training and the SME Community", Online: http://www.isbe.org.uk/ Training-and-the-SME-Community\#sthash.

jBidbXJj.dpuf, Received 2014. 
[16] Fraunhofer FOKUS, „Cloud-Computing in der öffentlichen Verwaltung - Chancen und Herausforderungen dynamischer IT-Dienstleistungen", Fraunhofer FOKUS, Berlin, 2010.

[17] Layo, I., "Cloud computing advantages for SMEs", http://cloudtimes.org/2013/09/18/cloud-computingadvantages-for-smes/, 18th Sept 2013, accessed on the 18th June 2014.

[18] Khalid, A., "Cloud Computing: Applying Issues in Small”, International Conference on Signal Acquisition and Processing, 2010.

[19] Carr, N., "The Big Switch: Re-Wiring the World, from Edison to Google", New York \& London: W.W. Norton, 2009.

[20] Hamburg, I., "Learning as a service - a cloud-based approach for SMEs", Service computation 2012: the Forth International Conference on Advanced Service Computing: pp. 53-57, 2012.

[21] Fernández, A., Peralta, D., Benítez, J.M. and Herrera, F., 'E-learning and educational data mining in cloud computing: an overview', Int. J. Learning Technology, Vol. 9, No. 1: pp. 25-52, 2014.

[22] Masud, A.H. and Huang, X., 'Esaas a new education soft ware model in e-learning systems', in Zhu, M. (Ed.): ICCIC 2011, Vol. 235 of CCIS, pp. 468-475, 2011.

[23] Ouf, S. and Nasr, M., 'Business intelligence in the cloud', IEEE 3rd International, 2011.Conference on Communication Software and Networks (ICCSN2011): pp. 650-655.

[24] Sulistio, A., Reich, C. and Doelitzscher, F., 'Cloud infrastructure and applications - cloudia', in Jaatun, M.G., Zhao, G. and Rong, C. (Eds.): CloudCom, Vol. 5931 of Lecture Notes in Computer Science: pp. 583-588, Springer, 2009.

[25] O'Brien, E. and Hamburg, I., "Supporting sustainable strategies for SMEs through training, cooperation and mentoring", Higher education studies 4/2: pp. 61-69, 2014. 